\title{
LISTENING TO MEMS: AN ACOUSTIC VIBROMETER
}

\author{
D.R. Yntema $^{1}$, J. Haneveld ${ }^{1}$, J.B.C. Engelen ${ }^{1}$, R.A Brookhuis ${ }^{1}$, \\ R.G.P. Sanders ${ }^{1}$, R.J. Wiegerink ${ }^{1}$, and M. Elwenspoek ${ }^{1,2}$ \\ ${ }^{1}$ TST group, MESA ${ }^{+}$and IMPACT Research Institutes, University of Twente, The Netherlands. \\ ${ }^{2}$ FRIAS, Albert-Ludwigs University, Albertstr. 19, 79104 Freiburg, Germany. \\ e-mail: d.r.yntema@utwente.nl
}

\begin{abstract}
A new way to characterize vibrating MEMS devices is presented. Using an acoustic particle velocity sensor the coupled sound field is measured, which is a measure for the movement of the MEMS device. We present several possible applications of this measurement method. It can be used as a read-out system for a mass flow sensor, and for characterization of in- and out-of-plane movements of MEMS devices. The method is an interesting alternative to laser scanning vibrometry due to its small size and low complexity; furthermore, it allows the user to 'listen' directly to MEMS devices.
\end{abstract}

\section{INTRODUCTION}

A vibrating structure in a medium generates movement of this medium. By approximation, the movement of the medium near a vibrating structure is equal to the movement of that structure [1]. The region where this occurs is called the very near field, which is dependent on the vibration frequency and the size (and shape) of the vibrating structure. For large structures $(>1 \mathrm{~cm})$ this method has already been validated [2]. When moving MEMS devices are to be characterized, the measurement must be done as close to the device as possible (typically $<1 \mathrm{~mm}$ ).

\section{Sound pressure versus Particle velocity}

In principle, it is possible to measure a sound field using ordinary pressure microphones, or alternatively, using particle velocity sensors. When the distance between sensor and sound source becomes very small, the particle velocity signal becomes increasingly larger compared to the sound pressure [3-4].

Also, the influence of background noise on the measured pressure signal is high. Measuring the particle velocity perpendicular to a non-moving plane, with only the small (MEMS) device moving, will yield the signal of the device, as well as some additional background noise. However, due to the boundary conditions (the non-moving plane), this noise level is much lower, compared to the noise level in the sound pressure signal. As a consequence, the signal to noise ratio is much higher when using a particle velocity sensor, when measuring in the very near field.
Additionally, the particle velocity allows for directional measurements, in contrast to a sound pressure measurement where this is not possible.

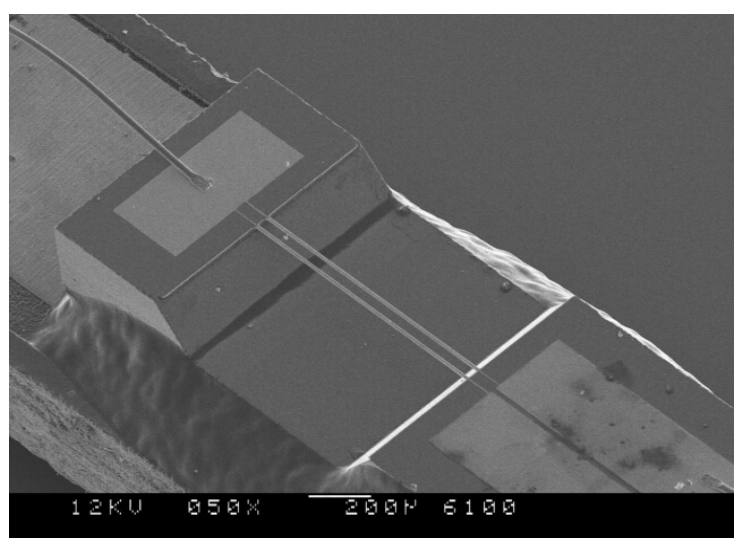

Figure 1: SEM image of the acoustic particle velocity sensor.

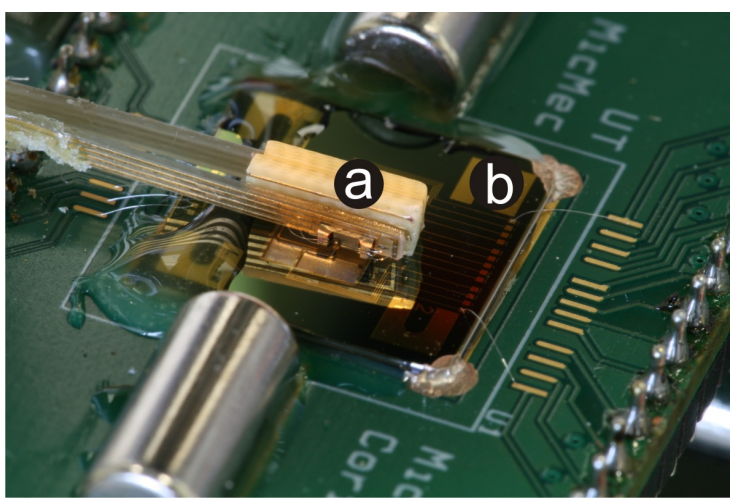

Figure 2: Photograph of the measurement setup. The probe with two particle velocity sensors (a), positioned over the device under test (in this case a micro Coriolis mass flow sensor) (b).

\section{Measurement set-up}

A sensor as shown in Fig. 1 is used to measure the acoustic particle velocity [5]. The sensor is mounted on a PCB, and attached to a micro probe manipulator. This assembly is then positioned over the structure to be measured (Fig. 2). An actuation signal is fed to the device under test and to the reference input of an analyzer. The sensor output is then coupled to the input channel of the analyzer. In this way a transfer function measurement between the excitation signal and the sensor response is obtained. 


\section{MEASUREMENT RESULTS}

Micro Coriolis mass flow sensor measurements.

Recently, our group has developed a micro Coriolis mass flow sensor, suitable for measuring liquid and gas mass flows in the $0-1 \mathrm{~g} / \mathrm{h}$ range [6-7].

A Coriolis type flow sensor consists of a vibrating tube, in which a liquid (or gas) mass flow is present. The moving (flowing) mass is forced to change its velocity due to the externally imposed vibration. This results in Coriolis forces that can be detected. Fig. 3 shows a schematic drawing of a Coriolis type sensor based on a rectangular tube shape. The tube is actuated in a "torsion mode" indicated by $\omega$. A mass flow $\Phi_{\mathrm{m}}$ inside the tube results in a Coriolis force $F_{c}$, which in turn induces a "flapping mode" vibration with an amplitude proportional to the mass flow.

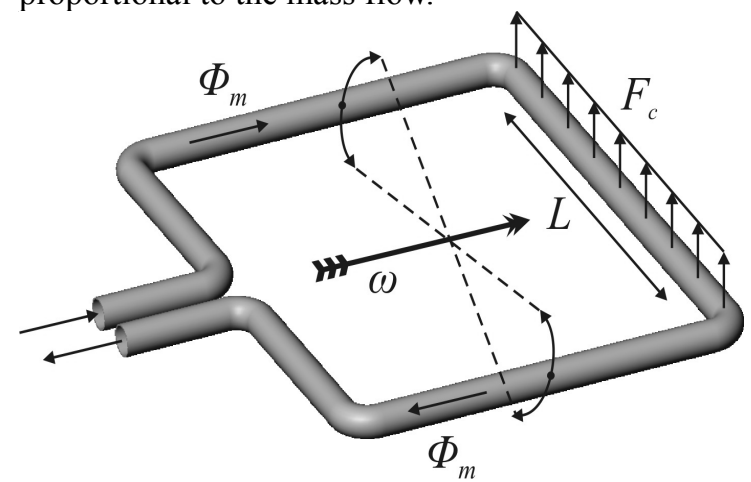

Figure 3: Rectangle-shaped Coriolis flow sensor ( $\omega$ $i$ the torsion mode actuation vector, $F_{c}$ indicates the Coriolis force due to mass flow).

The micro Coriolis mass flow sensor can be read out using a laser scanning vibrometer [6], or by onchip capacitive read-out structures [7]. For the work presented in this paper, two particle velocity sensors were used, mounted back-to-back as shown in Fig. 2.

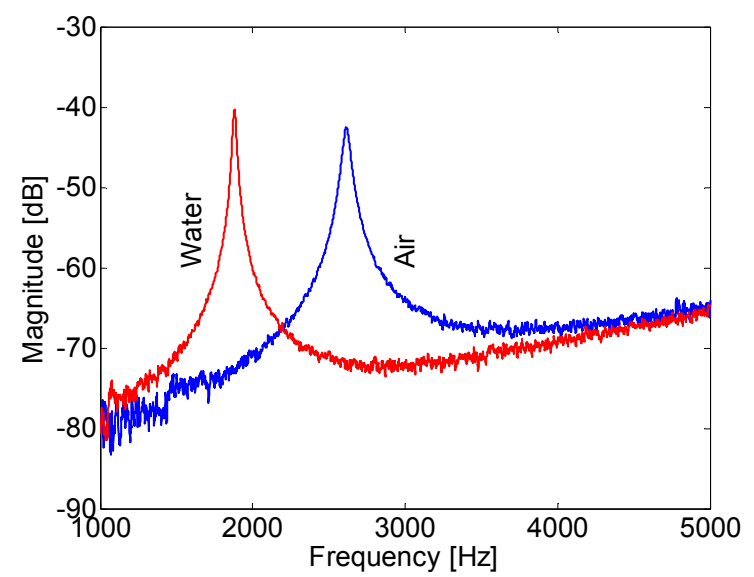

Figure 4: Out-of-plane resonance frequency of the micro Coriolis mass flow sensor when filled with water and air, measured using a particle velocity sensor.
The vibration amplitude of the Coriolis tube is in the order of a few microns, at a resonance frequency of $2.6 \mathrm{kHz}$ when filled with air, and 1.8 $\mathrm{kHz}$ when filled with water, see Fig. 4.

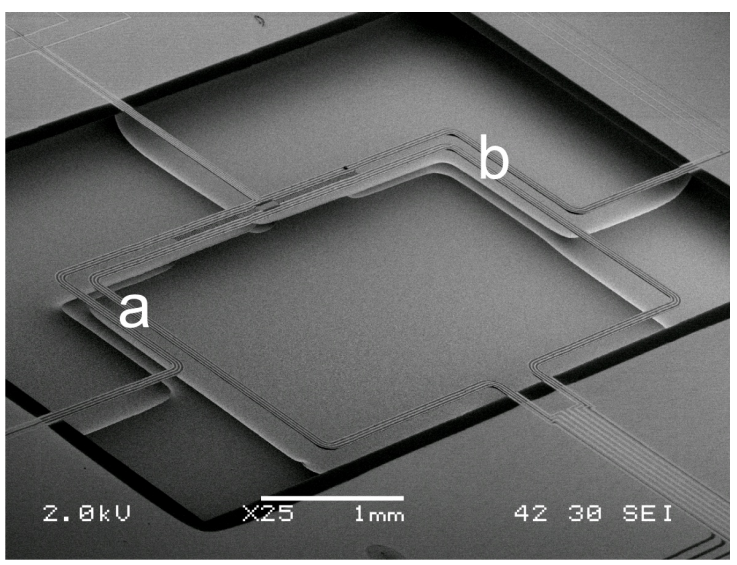

Figure 5: SEM image of the micro Coriolis mass flow sensor.

In order to perform a mass flow measurement, the oscillating rectangular structure is monitored at two corners (points ' $a$ ' and ' $b$ ' in Fig. 5). When mass flow is applied, the phase difference between the signals originating from these points is a direct measure for the mass flow rate. Similar to the sensor being read out by laser scanning vibrometry and capacitive structures, we were able to accurately measure mass flow rates up to $1.4 \mathrm{~g} / \mathrm{hr}$ (Fig. 6).

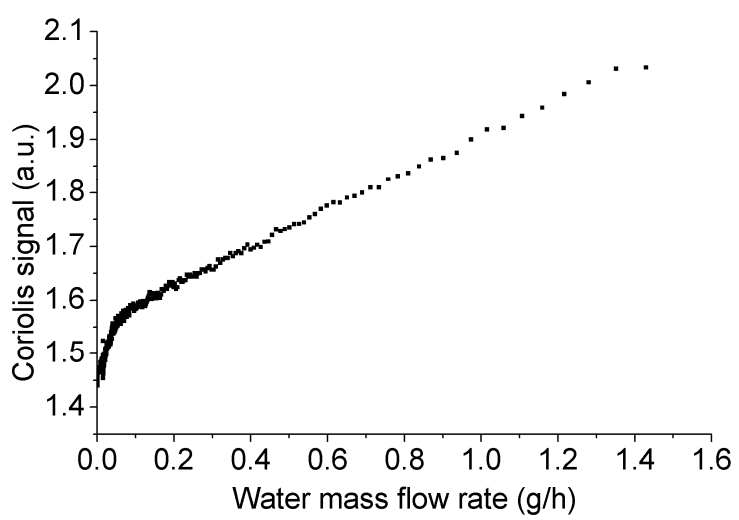

Figure 6: Liquid mass flow measurement with 0-1.4 $\mathrm{g} / \mathrm{hr}$ of water and two particle velocity probes as shown in Fig. 2.

An interesting, but yet to be explained phenomenon is observed for mass flows lower than $0.1 \mathrm{~g} / \mathrm{h}$. The graph is not linear in this region, whereas the Coriolis force itself is linear with the mass flow. For mass flows larger than $0.1 \mathrm{~g} / \mathrm{h}$, the signal is as expected. 
Comb drive in-plane measurements.

A comb drive is a common MEMS actuator and sensor, using electrostatic forces to generate or measure motion in many MEMS devices. However, since its motion is in-plane, standard laser vibrometry cannot be used to characterize its movement. A standard method for characterizing in-plane movement, is using a microscope setup with a camera to take images of the device synchronized with the applied signal. To overcome the frame rate limit of the camera, stroboscopic illumination is used. When the measurement is completed, each image is analyzed by image processing software with motion estimation to determine the movement of the device, or parts thereof. The frequency response can be determined either by measuring the step response and taking its Fourier transform, or by applying a series of sine waves with different frequencies and measure the corresponding displacements. Typically many images are required to determine the resonance frequency accurately, and complete measurements take on the order of tens of minutes.

Alternatively, measurements with a particle velocity sensor have a lower spatial resolution, and absolute displacement measurements are not straightforward, but a single measurement only takes a couple of seconds. So, if knowledge of the frequency response is desired, a particle velocity sensor can provide an accurate value in a short amount of time.

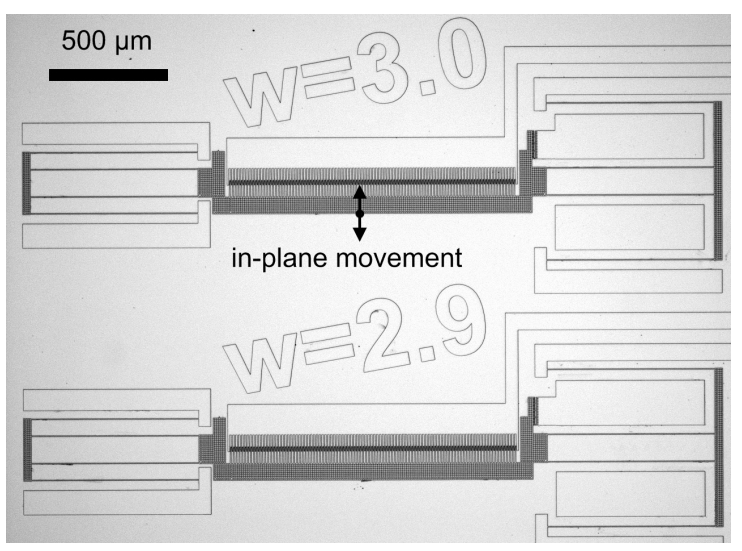

Figure 7: Microscope image showing two comb drives. The darker parts of the comb drive are the moving translators.

The frequency response of an in-plane moving comb drive (Fig. 7) was measured using both a Polytec MSA400 laser scanning vibrometer, and a particle velocity sensor. The comb drive, discussed elsewhere in these proceedings [8] is a $25 \mu \mathrm{m}$ high, $1.3 \mathrm{~mm}$ long moving 'wall', suspended $1 \mu \mathrm{m}$ above the substrate. Measurements with a particle velocity sensor, applying a white noise signal, show an inplane resonance frequency of $1.81 \mathrm{kHz}$. The laser scanning vibrometer measurements confirm this value, and show a displacement amplitude of $2 \mu \mathrm{m}$ at resonance using the same excitation voltage. This corresponds to a movement velocity of $23 \mathrm{~mm} / \mathrm{s}$.

Fig. 8 shows the frequency response of the comb drive as measured by the particle velocity sensor. At low frequencies, the displacement speed is too low for the sensor to detect, but the resonance frequency of the beam is clearly identified. At higher frequencies, the response seems to increase further. This is not due to the actual displacement of the comb drive but due to electrical coupling of signals. This problem is solvable in the future by better electric shielding of the sensor.

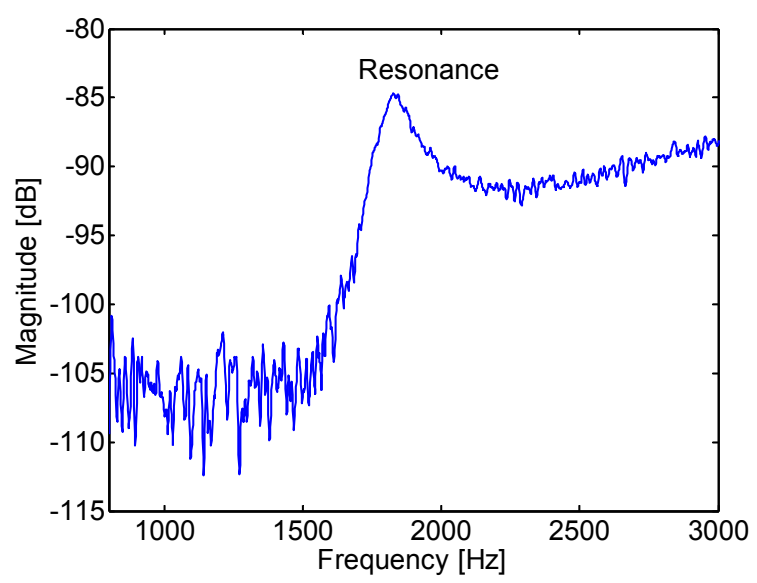

Figure 8: In-plane resonance frequency of the comb-drive structure.

\section{Micro mirror characterization.}

Characterization of the fundamental resonance frequency and higher order modes gives useful information on the mechanical properties of micromirrors [9]. Since this involves out-of-plane movement, this task can be performed with a laser scanning vibrometer. The same measurement can also be performed using a particle velocity sensor, for a quick characterization of the modes.

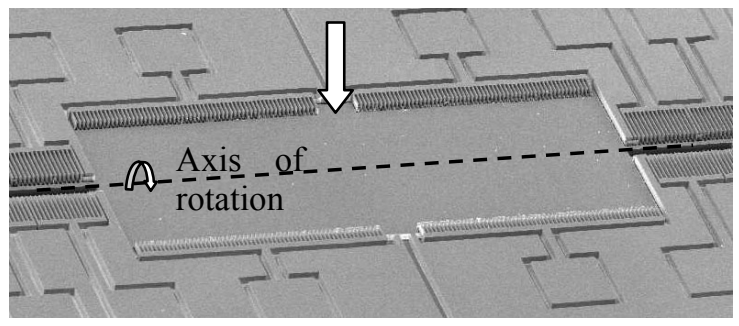

Figure 9: Micro mirror device, fabricated in the $\mathrm{MESA}^{+}$Clean Room. The particle velocity is measured above the rotating endpoint (indicated by the large arrow). 
For a good comparison, both measurement methods are performed simultaneously in one measurement setup. The displacement and the particle velocity are both measured above the rotating endpoint of the mirror surface, see Fig. 9.

The particle velocity sensor is positioned such that it is sensitive for particle velocity in the direction normal to the mirror surface.

The micro mirror is electrostatically actuated by applying a frequency sweep from $10 \mathrm{~Hz}$ to $10 \mathrm{kHz}$. The result of this measurement is shown in Fig. 10. From the frequency response, two modes can be distinguished. The first mode is the desired torsion mode around the indicated axis $(225 \mathrm{~Hz})$, the second is the out-of-plane sliding mode at $4.0 \mathrm{kHz}$. For the measurement shown in Fig. 10, the out-ofplane displacement of the mirror is $+/-40 \mathrm{~nm}$ for the torsion mode and $+/-150 \mathrm{pm}$ for the out-ofplane sliding mode, measured by a laser scanning vibrometer (Polytec MSA400). Due to the directional sensitivity of the particle velocity sensor other higher order modes which involve in-plane movement of the mirror surface are not visible in the frequency response.

For large angle deflections in the torsion mode, the laser vibrometer runs into problems. In this particular case, the rotational angle of the structure is so large $\left(>1.5^{\circ}\right)$ that the reflected beam does not even enter the objective. Positioning the particle velocity sensor nearby one end of the micro mirror does give a well-defined frequency response.

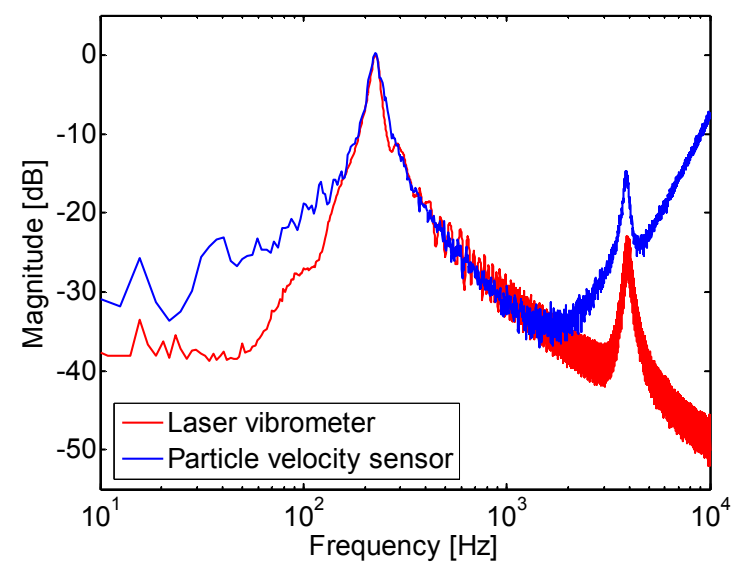

Figure 10: Micro mirror resonance measurements using both a particle velocity sensor and a laser scanning vibrometer (normalized at the first resonance frequency).

\section{CONCLUSIONS}

A method to acoustically characterize moving MEMS devices is presented. Instead of a laser vibrometer a particle velocity sensor is used. The method proved to be versatile and useful for both out-of-plane and in-plane measurements. Although absolute displacement measurements are less accurate, numerous experiments do not require this and the method can well be used for characterisation of MEMS devices.

\section{ACKNOWLEDGEMENTS}

The authors wish to thanks the Dutch Technology Foundation (STW), the Dutch MicroNed program, and the Dutch MEMSLand program for financial support.

\section{REFERENCES}

[1] R. Raangs, "Exploring the use of the microflown", $\mathrm{PhD}$ thesis, University of Twente, Enschede, The Netherlands, 2005.

[2] H-E. de Bree, V.B. Svetovoy, R. Raangs, R. Visser, "The very near field - Theory, simulations, and measurements of sound pressure and particle velocity in the very near field", Proceedings of the ICSV11, St. Petersburg, Russia, 2004.

[3] H-E. de Bree, W.F. Druyvesteyn, "A particle velocity sensor to measure the sound from a structure in the presence of background noise", Forum Acousticum, Budapest, Hungary, Aug. 29 - Sep. 2, 2005.

[4] J.W. Wind, D.R. Philippens, A. de Boer, "Near Source Acoustical Particle Velocity Measurements with Ambient Noise", Proceedings of IMAC-XXV, Orlando, Fl, Feb. 19-22, 2007.

[5] H-E. de Bree, "The Microflown: an acoustic particle velocity sensor", Acoustics Australia, vol. 31, No. 3, pp. 91-94, 2003.

[6] J. Haneveld, T.S.J. Lammerink, M. Dijkstra, H. Droogendijk, M.J. de Boer, R.J. Wiegerink, "Highly sensitive micro Coriolis mass flow sensor", Proceedings of IEEE MEMS 2008, Tucson, Az, Jan. 13-17, 2008, pp. 920-923.

[7] J. Haneveld, T.S.J. Lammerink, M.J. de Boer, R.J. Wiegerink, "Micro Coriolis mass flow sensor with integrated capacitive readout", Proceedings of IEEE MEMS 2009, Sorrento, Italy, Jan. 25-29, 2009, pp. 463-466.

[8] J. B. C. Engelen, L. Abelmann, M. C. Elwenspoek, "Optimized comb drive finger shape for shock-resistant actuation", Proceedings of IEEE MEMS 2010, Hong Kong, China, Jan. 24-28, 2010.

[9] H. Urey, Cihan Kan, W.O. Davis, "Vibration mode frequency formulae for micromechanical scanners", Journal of Micromechanics and Microengineering, vol. 15, pp. 1713-1721, 2005. 